\title{
Topical RNAi for Sustainable Animal Health ${ }^{\dagger}$
}

\author{
Karishma T. Mody 1, Bing Zhang 2, Xun Li 1 , Ritesh Jain 1, Peng Li ' ${ }^{3}$, Peter James ${ }^{1}$, \\ Timothy J. Mahony ${ }^{1}$, Zhiping $\mathrm{Xu}^{3}$ and Neena Mitter ${ }^{1, *}$ \\ 1 Queensland Alliance for Agriculture and Food Innovation, St. Lucia, Qld 4072, Australia; \\ k.mody@uq.edu.au (K.T.M.); xun.li@uq.net.au (X.L.); r.jain1@uq.edu.au (R.J.); p.james1@uq.edu.au (P.J.); \\ t.mahony@uq.edu.au (T.J.M.) \\ 2 Animal Science, Queensland Department of Agriculture and Fisheries, Ecosciences Precinct, Dutton Park, \\ Qld 4102, Australia; bing.zhang@daf.qld.gov.au \\ 3 Australian Institute for Bioengineering and Nanotechnology, St. Lucia, Qld 4072, Australia; \\ p.li3@uq.edu.au (P.L.); gordonxu@uq.edu.au (Z.X.) \\ * Correspondence: n.mitter@uq.edu.au \\ + Presented at the third International Tropical Agriculture Conference (TROPAG 2019), Brisbane, Australia, \\ 11-13 November 2019.
}

Published: 7 April 2020

\begin{abstract}
Animal health measures mainly rely on vaccination or chemical control for major pests and pathogens, causing issues of residue, toxicity and development of resistance. For example, control of Sheep flystrike and lice-infestation affecting the Australia's sheep/wool industry (>3.5 B) have developed resistance to nearly all control chemicals used in the past. Topicals RNAi provides an innovative clean-green, non-toxic, environmentally sustainable biological control solution. Biodegradable clay particles as carriers can be used to deliver double stranded RNA (dsRNA), the key trigger molecule of RNA interference pathway. As an early proof of concept, we investigated the stability dsRNA loaded on two types of Clay particles: Clay1 (releases dsRNA under acidic conditions) and Clay2 (releases dsRNA under alkaline conditions) on cattle hide. Cattle skin was treated with Cy3 labelled dsRNA alone and Cy3 labelled dsRNA loaded on Clay1 or Clay2. The skin samples treated with the $\mathrm{Cy} 3$ formulations were imaged using confocal microscopy. Once imaged, the skin samples were washed and stored at room temperature for 5 days, later the samples were re-imaged to detect the fluorescent signal (Figure 1). The dsRNA loaded on clay particles was stable unlike naked Cy3-dsRNA which degraded and was not visible after washing. This increased inherent stability of the dsRNA molecules, combined with the environmental stability afforded by the Clay particles, offers promise to provide a sustainable solution for animal health. Topical RNAi can reduce reliance on trade withholding periods of meat/wool without chemical residues, enhance animal welfare and increase production of premium quality meat/wool, improve export potential, competitiveness and long-term profitability of livestock industry.
\end{abstract}

Keywords: RNAi; clay particles; sheep flystrike; lice-infestation; animal health; sustainable solution 


\section{A) Cy3 labelled dsRNA}
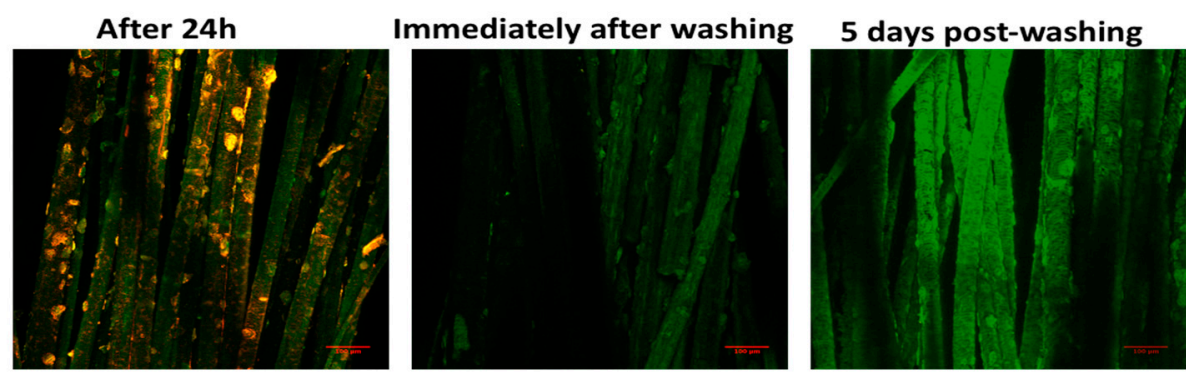

B) Cy3 labelled dsRNA with Clay 1

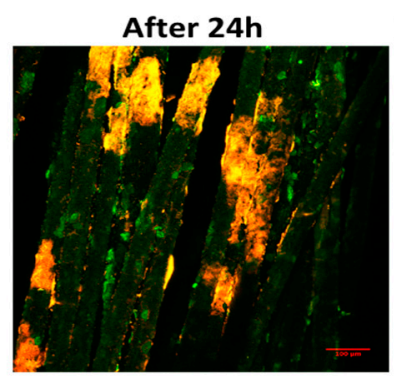

Immediately after washing
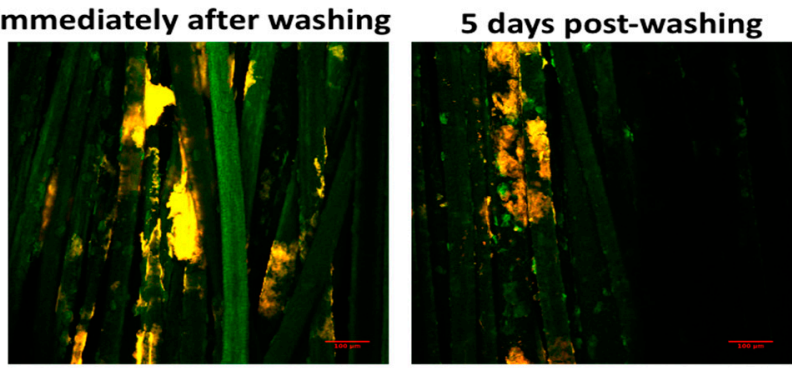

\section{C) Cy3 labelled dsRNA with Clay 2}
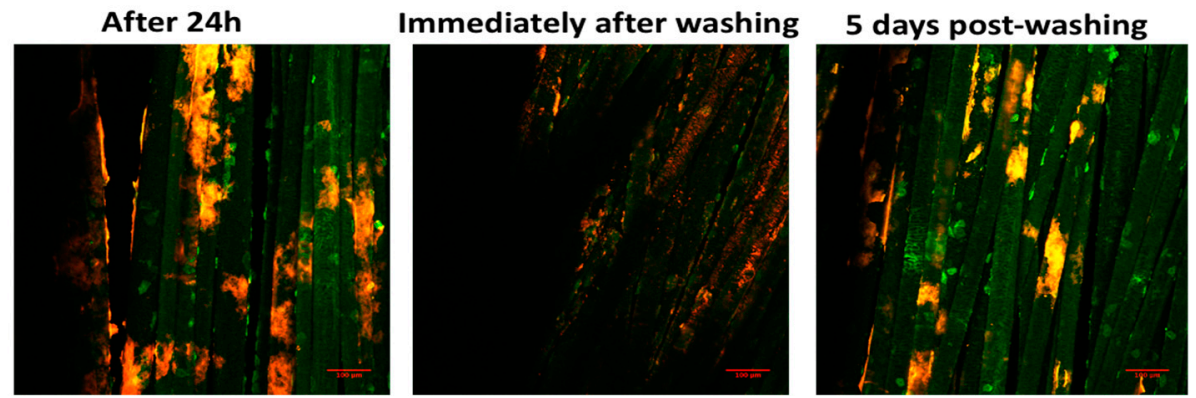

Figure 1. Confocal image of cattle skin treated with Cy3-dsRNA/Cy3-dsRNA loaded on Clay1 or Clay2. Samples imaged $24 \mathrm{~h}$ after application of treatment; immediately after washing and 5 days post-washing. (A) Cy3 labelled dsRNA; (B) Cy3 labelled dsRNA with Clay1; and (C) Cy3 labelled dsRNA with Clay2. The confocal microscopy was performed at the Australian Cancer Research Foundation (ACRF)/Institute for Molecular Bioscience Cancer Biology Imaging Facility, which was established with the support of the ACRF.

Conflicts of Interest: The authors declare no conflict of interest.

(c) 2020 by the authors. Licensee MDPI, Basel, Switzerland. This article is an open access article distributed under the terms and conditions of the Creative Commons Attribution (CC BY) license (http://creativecommons.org/licenses/by/4.0/). 